\title{
Valoración del Podcasting en la enseñanza clínica en el área de odontología restauradora
}

\author{
Valuation of the use of Podcasting for clinical education in the area of restorative dentistry \\ Miguel Saravia ${ }^{1, \mathrm{a}}$, Francisco Orejuela ${ }^{1, \mathrm{~b}}$, Mary Fukuhara ${ }^{1, \mathrm{c}}$
}

\begin{abstract}
RESUMEN
Objetivo: Describir la valoración del podcasting en el proceso de aprendizaje en estudiantes de odontología en una universidad privada. Material y métodos: La investigación fue de naturaleza cuantitativa, descriptiva, no experimental. La muestra estuvo constituida por 47 estudiantes de una facultad de odontología de la ciudad de Lima, Perú. Para la experiencia se tomaron artículos en PDF de las clases teóricas cuyo contenido fueron grabados usando unos audífonos convencionales y el aplicativo de Spreaker REC ${ }^{\circledR}$, aplicativo gratuito que se instaló en un teléfono inteligente Iphone 8, se procedió a grabar producir y editar en un tiempo menor a 15 minutos el artículo en PDF usando dicho aplicativo. Ulteriormente se envió al grupo de WhatsApp ${ }^{\circledR}$ el link para que pudieran acceder al audio. Este podía ser repetido tantas veces como el estudiante lo desee escuchar. Se realizaron tres Podcast. Se recolectó la información mediante encuestas a través de un cuestionario utilizado la escala tipo Licker. Resultados: El 70,22\% de los encuestados consideraron que el podcast compartido en el grupo de WhatsApp ${ }^{\circledR}$ tuvo un a alta y muy alta contribución en su desempeño clínico. Se encontró que el podcast fue considerado como una herramienta de fácil consulta $(95,74 \%)$, facilitan el aprendizaje $(75,74 \%)$ y contribuye a aprender en menos tiempo (93,62\%). E1 62,07\% lo consideró un buen recurso para contenidos teóricos. Conclusiones: Los estudiantes consideran que el podcast es una herramienta que contribuye a su aprendizaje, sobretodo es muy útil para el aprendizaje de contenidos teóricos.
\end{abstract}

PALABRAS CLAVE: Podcasting, educación superior, educación dental.

\begin{abstract}
SUMMARY
Objective: To describe the valuation of podcasting as a tool in the learning process by dental students at a private university. Material and methods: The research was quantitative, descriptive, and non-experimental. The sample consisted of 47 students from a dental school. For the experience, articles in PDF format from the theoretical classes were recorded using conventional headphones and the application Spreaker REC ${ }^{\circledR}$, a free application that was installed in a smartphone Iphone 8. The article in PDF format was processed, recorded and edited in less than 15 minutes using said application. Subsequently, the link was sent to the WhatsApp ${ }^{\circledR}$ group so the students could access the audio. The podcast could be repeated as many times as the student desired. Three podcasts were made. The information was collected through surveys using a Licker-type survey. Results: The $70.22 \%$ of the respondents considered that the shared podcasts in the WhatsApp ${ }^{\circledR}$ group had a high and very high contribution to their clinical performance. It was found that the podcasts were of easy consultation (95,74\%), facilitated learning $(75,74 \%)$ and contributed to a shorter learning time $(93,62 \%)$. Overall, $62,07 \%$ considered podcasting a good resource for the theoretical content. Conclusions: Students consider that podcasting is a tool that contributes to their learning, particularly as a very useful vehicle for learning theoretical content.
\end{abstract}

KEY WORDS: Podcasting, higher education, dental education

Facultad de Estomatología, Universidad Peruana Cayetano Heredia. Lima, Perú

Docente Principal, Cirujano Dentista, Maestría en Estomatología, Doctor en Estomatología

Docente Auxiliar, Cirujano Dentista.

Docente Asociado, Cirujano Dentista, Maestría en Estomatología, Maestría en Educación con mención en Docencia e Investigación en Educación Superior 


\section{INTRODUCCIÓN}

Un podcast es un archivo de audio o video a los que podemos suscribirnos y que pueden ser descargados en una computadora o en un dispositivo portátil de audio incluyendo un teléfono celular inteligente. El término podcasting hace referencia a la acción de crear estos archivos de sonido. Las potencialidades del podcast para la enseñanza son numerosas sobre todo en la educación superior (1).

Los podcasts se pueden descargar a reproductores de medios portátiles que se pueden llevar a cualquier lugar, lo que brinda la posibilidad de experiencias de aprendizaje "en cualquier momento y en cualquier lugar" (M-learning). Asimismo, si se implementan de manera efectiva, podrían constituirse en herramientas que contribuirían a mejorar las experiencias de aprendizaje de los estudiantes, clínicos y pacientes, y profundizar los niveles de participación y colaboración de los alumnos en entornos de aprendizaje digital $(2,3)$.

Al revisar la literatura hemos encontrado que el podcasting es una metodología usada en varias instituciones con fines académicos y aplicados con singular éxito para las carreras de ciencias de la salud y otras ciencias. Estas tecnologías propician el cambio hacia un paradigma educativo caracterizado, entre otros; por la independencia del proceso de enseñanza-aprendizaje de espacios físicos o temporales, así como por un mayor control de los estudiantes sobre su propio aprendizaje $(4,5,6,7)$.

En otro estudio realizado por McCann, Schneiderman, Hinton, en estudiantes de odontología e higienistas dentales, sobre sus preferencias para la enseñanza y aprendizaje a través del uso de recursos electrónicos, se encontró que los estudiantes están a favor de estos recursos pero como complemento de las conferencias dadas por parte de los profesores, manifestaron que estos materiales mejorarían mucho el aprendizaje, como conferencias $(59 \%)$, videos clínicos $(54 \%)$ y podcast $(45 \%)(8)$.

El presente estudio tuvo como objetivo describir la valoración del podcasting en el proceso de aprendizaje en estudiantes de odontología en una universidad privada de Lima, Perú.

\section{MATERIAL Y MÉTODOS}

La investigación fue de naturaleza cuantitativa, descriptiva, no experimental. La muestra fue no probabilística y se empleó una selección muestral por conveniencia. Fueron un total de 47 estudiantes de un total de 57 estudiantes del tercer año de la carrera de estomatología del curso de Clínica integral del Adulto II, quienes aceptaron participar en este estudio, para lo cual se tomaron artículos en PDF de las clases teóricas. Posteriormente, se grabaron usando un micrófono convencional usando el aplicativo de Spreaker $\mathrm{REC}^{\circledR}$, aplicativo gratuito que se instaló en un teléfono inteligente Iphone 8 , se procedió a grabar producir y editar en un tiempo menor de 15 minutos el artículo de PDF. Ulteriormente, se envió al chat del grupo de estudiantes el link para que pudieran acceder al audio. Este podía ser repetido tantas veces como el alumno lo desee escuchar. Se realizaron tres Podcast.

Para evaluar la experiencia se tomó una encuesta a través de un cuestionario utilizando la escala tipo Liker validada entre pares. Los datos obtenidos de los cuestionarios fueron procesados al programa Microsoft Excel (Microsoft Office 2007); para el análisis se exportó la base de datos al programa STATA ${ }^{\circledR}$ versión 15. Para el analisis descriptivo se emplearon tablas de frecuencias absolutas y relativas.

\section{RESULTADOS}

El 70,22\% de los encuestados consideraron que el podcast compartido en el grupo de WhatsApp ${ }^{\circledR}$ tuvo un a alta y muy alta contribución en su desempeño clínico (tabla 1).

En cuanto al motivo por el cual accedieron a los podcasts, el 35,63\% manifestaron que accedieron como una forma de adquirir conocimiento sin necesidad de leer, el 34,48\% porque le sirvió para repasar para los exámenes y un $29,89 \%$ accedió a este material para buscar información específica (tabla 2).

Se encontró que el podcast es de fácil consulta $(95,74 \%)$, facilitan el aprendizaje $(75,74 \%)$ y contribuye a aprender en menos tiempo $(93,62 \%)$ (tabla 3$)$, y el $62,07 \%$ lo consideró un buen recurso para contenidos teóricos (tabla 4). 
Tabla 1. Medidas en que el material educativo (audios) ha contribuido a mejorar tu desempeño clínico

\begin{tabular}{lcc}
\hline \hline & $\mathrm{n}$ & $\%$ \\
\hline Ninguna Contribución & 1 & 2,13 \\
Baja Contribución & 1 & 2,13 \\
Moderada Contribución & 12 & 25,53 \\
Alta Contribución & 19 & 40,43 \\
Muy Alta Contribución & 14 & 29,79 \\
Total & 47 & 100 \\
\hline \hline
\end{tabular}

Tabla 2. Motivo por el cual accedieron al Podcast (audios)

\begin{tabular}{lcc}
\hline \hline & $\mathrm{n}$ & $\%$ \\
\hline Buscar información específica & 26 & 29,89 \\
Conocimiento sin leer & 31 & 35,63 \\
Repasar para exámenes & 30 & 34,48 \\
Total & 87 & 100,00 \\
\hline
\end{tabular}

Tabla 3. Frecuencia de la Utilidad de los Podcast

\begin{tabular}{lcccc}
\hline \hline & $\begin{array}{c}\text { Son de fácil } \\
\text { consulta }\end{array}$ & $\begin{array}{c}\text { Aprender } \\
\text { en menos } \\
\text { tiempo }\end{array}$ & $\begin{array}{c}\text { Facilitan el } \\
\text { aprendizaje }\end{array}$ & $\begin{array}{c}\text { Presenta información } \\
\text { académicamente } \\
\text { relevante }\end{array}$ \\
\hline Completamente en desacuerdo & $1(2,13)$ & $2(4,26)$ & $1(2,13)$ & $2(4,26)$ \\
En desacuerdo & $1(2,13)$ & $1(2,13)$ & $1(2,13)$ & $2(4,26)$ \\
De acuerdo & $21(44,68)$ & $25(53,19)$ & $25(53,19)$ & $26(55,32)$ \\
Completamente de acuerdo & $24(51,06)$ & $19(40,43)$ & $20(42,55)$ & $17(36,17)$ \\
Total & $47(100,0)$ & $47(100,0)$ & $47(100,0)$ & $47(100,0)$ \\
\hline
\end{tabular}

Tabla 4. Contenidos donde los Podcast se perciben que podrían mejorar el aprendizaje

\begin{tabular}{lcc}
\hline & $\mathrm{n}$ & $\%$ \\
\hline Endodoncia & 7 & 24,14 \\
Oclusión & 1 & 3,45 \\
Colocación de Postes & 1 & 3,45 \\
Blanqueamiento Dental & 1 & 3,45 \\
Contenidos teóricos & 18 & 62,07 \\
Medicina Bucal & 1 & 3,45 \\
Total & 29 & 100 \\
\hline
\end{tabular}




\section{DISCUSIÓN}

En el estudio de Kapoor, Catton y Khalil, encontraron que la mayoría de los estudiantes consideraron que los podcasts eran claros, de una longitud adecuada, orientados al nivel académico correcto y que proporcionaban un buen método de aprendizaje. Sin embargo, en relación con la preferencia de los podcasts sobre los métodos de aprendizaje convencionales las opiniones estuvieron divididas, identificándose que los podcasts realizados en un estilo de entrevista con una voz atractiva e imágenes servirían para mantener la motivación en los estudiantes. Este resultado podría considerarse semejante a nuestro estudio ya que el $62,07 \%$ encontró que los podcasts serían útiles para contenidos teóricos (9).

El $95,74 \%$ de los estudiantes encuestados consideraron que el podcast era un material de fácil consulta, resultados semejantes al hallado en el estudio realizado por Kalludi, Punja, Pai, et al., quienes reportaron que el 91,3\% de los estudiantes consideraron que los podcasts eran útiles, ya que podían escuchar el contenido de las conferencias repetidamente y en el momento que tuvieran disponibilidad de tiempo y en cualquier lugar (10).

Kalludi, Punja, Rao y Dhar en su investigación que tuvo cuyo objetivo fue evaluar la actitud de los estudiantes de primer año de odontología con respecto a la complementación de video podcast y evaluar la eficacia del video podcast como ayuda para la enseñanza en comparación con la lectura de libros de texto, encontraron que los estudiantes que se sometieron a la sesión de podcast de video obtuvieron mejores resultados en la evaluación en comparación con los estudiantes que se sometieron solo a la lectura de un libro de texto. Esto demuestra una ventaja de los podcasts de video sobre la lectura de libros de texto. La mayoría de los estudiantes aceptó los beneficios de la suplementación de video podcast (11).

Schreiber, Fukuta y Gordon, en su estudio que tuvo como objetivo comparar la efectividad de una conferencia en vivo y el podcast en un grupo de estudiantes, encontrando que los sujetos disfrutaron de la conveniencia del video podcast y la capacidad de tenerlo, revisarlo y repetirlo, pero lo encontraron menos atractivo como método de enseñanza. Expresaron una clara preferencia por el formato de la conferencia en vivo, además no se halló diferencia significativa en cuanto a las respuestas a las preguntas aplicadas luego de la sesión de aprendizaje (12).

Walmsley, Lambe, Perryer y Hill en su investigación que tuvo como objetivo evaluar la reacción de los estudiantes de los cursos clínicos al uso de Podcasts para la enseñanza de pregrado encontraron que los podcasts cortos son un medio útil para entregar materiales de aprendizaje a los estudiantes, ofreciéndoles flexibilidad y están acorde con las características de los estudiantes de esta generación de descargar materiales para utilizarlos cuando lo deseen lo que coincide con lo hallado en nuestro estudio (13).

Lowman, en un estudio realizado para indagar sobre el impacto de los podcasts y vodcasts (similar al podcasting sólo que en vez de tener audio tiene audio e imagen) a los que pudieron acceder a través de un iPod para el aprendizaje de vocabulario en estudiantes de educación básica, se halló que en promedio, los estudiantes aprendieron cuatro de cada nueve palabras enseñadas sin importar la asignación de grupo. El grupo de vodcast aprendió significativamente más palabras en comparación con el grupo de podcast a nivel receptivo y expresivo. Los comentarios de los estudiantes indicaron que los iPods deben usarse para enseñar y revisar palabras (14).

Guze, considera que algunas tecnologías como podcasts y videos, dispositivos móviles con aplicaciones, videojuegos, simulaciones (capacitadores a tiempo parcial, simuladores integrados, realidad virtual) y dispositivos portátiles son algunas de las técnicas disponibles para abordar el cambiante entorno educativo (15).

Finalmente es importante considerar que para la implementación de un podcast es necesario un proceso de reflexión y planificación, procesos que concluyen con una evaluación, en ese sentido es que siendo el podcast un recurso didáctico, para su elaboración es necesario considerar a quién está dirigido, qué objetivo tiene y cómo se va a utilizar. El podcast puede ser creado tanto por el profesor como por los estudiantes $(16,17)$

\section{CONCLUSIONES}

El podcast es considerado una herramienta muy útil por los estudiantes en su proceso de aprendizaje, so- 
bre todo para el aprendizaje de los contenidos teóricos, el podcast permite al estudiante acceder las veces que le resulte necesario al material de estudio y sobretodo es una herramienta de aprendizaje acorde con las tecnologías utilizadas por esta generación de estudiantes.

Los estudiantes consideran que el podcast contribuyó a mejorar su desempeño clínico.

Este estudio presenta algunas limitaciones como el tamaño de muestra la cual podría ampliarse, asimismo habiendo utilizado una encuesta tipo Licker los datos recogidos están limitados pudiendo subsanarse a través de una entrevista para recoger datos cualitativos.

\section{Correspondencia}

Mary Fukuhara

Correo electrónico: Mary.fukuhara@upch.pe

\section{REFERENCIAS BIBLIOGRÁFICAS}

1. Solano I, Sanchez M. Aprendiendo en cualquier lugar: el podcast educativo. Pixel-Bit. Revista de Medios y Educación. 2010;36:125-139.

2. Kamel M, Maramba1 I, Wheeler S. Wikis, blogs and podcasts: a new generation of Web-based tools for virtual collaborative clinical practice and education MC Medical Education. 2006, 6 (41): 1-8.

3. Sigüenza J, Montánchez M, Palta N. La tutoría académica y la herramienta web 2.0: podcast, en la educación superior. Revista Killkana Sociales. 2018; 2(2): 39-46.

4. Jham B, Duraes G, Strassler H, Sensi L. Joining the podcast revolution. J Dent Educ. 2008; 72 (3):278-81.

5. Horvath Z, O' Donnell J, Jhonson L Karimbux N, Shule C, Spallek H. Use of lecture recordings in dental education: assesment of status quo and reomendations J Dent Educ. 2013; 77 (11):1431-42.

6. Allen K, Katz R. Comparative use of podcast vs lectura transcripts as learning aids for dental students J Dent Educ. 2011; 75 (6):817-22.

7. Kalludi S, Punja, Pai K, Dhar M, Efficacy and perceived utility of podcats as supplementary teaching aid among first-year dental students. Austrlas Med J. 2013; 6 (9):450-7.

8. McCann A, Schneiderman E, Hinton R. E-Teaching and learning preferences of dental and dental hygiene students. Journal of Dental Education. 2010; 74 (1): 65-78.

9. Kapoor S, Catton R, Khalil H. An evaluation of medical student-led podcasts: what are the lessons learnt? Advances in Medical Education and Practice. 2018; (9): 133-138.
10. Kalludi S, Punja D, Pai K, Dhar M. Efficacy and perceived utility of podcasts as a supplementary teaching aid among first-year dental students. AMJ. 2013; 6(9):450-457.

11. Kalludi S, Punja D, Raghavendra R, Murali D. Is video podcast supplementation as a learning aid beneficial to dental students? Journal of Clinical and Diagnostic Research. 2015. 9(12): 4-7.

12. Schreiber B, Fukuta J, Gordon F. Live lecture versus video podcast in undergraduate medical education: A randomised controlled trial. BMC Medical Education. 2010; 10 (68): 1-6.

13. Walmsley A, Lambe C, Perryer D, Hill K. Podcasts an adjunct to the teaching of dentistry. British Dental Journal. 2009; 206 (3):157-160.

14. Lowman J. Exploring the use of podcasts and vodcasts: Multimedia tools for word. Learning Comput Sch. 2014;31(4):251-270.

15. Guze P. Using technology to meet the challenges of medical education. Trans Am Clin Climatol Assoc. 2015; 126: 260-270.

16. Piñeiro $T$. Los podcast en la educación superior. Hacia un paradigma de formación intersticial. Revista Iberoamericana de Educación. 2012; 58(1):1-12.

17. Pérez-de-Pedro J. Podcasting: tú tienes la palabra, Madrid: Bubok; 2010.

Recibido: 20-12-19

Aceptado: 15-04-20 